\title{
Non-Human Leukocyte Antigen Antibody-Mediated Lung Transplant Rejection: The Other Anti-A
}

\author{
Anthony D. Vinson, DO, ${ }^{1}$ Reinaldo Rampolla-Selles, MD, ${ }^{2}$ E. Shannon Cooper, MD, JD, ${ }^{3}$ \\ Caroline R. Alquist, MD, $\mathrm{PhD}^{3}$ \\ ${ }^{1}$ Department of Pathology, Louisiana State University Health Sciences Center, New Orleans, LA ${ }^{2}$ Multi-Organ Transplant Institute, \\ Ochsner Clinic Foundation, New Orleans, LA ${ }^{3}$ Department of Pathology, Ochsner Clinic Foundation, New Orleans, LA
}

Background: Acute rejection of lung allografts is an important contributor to morbidity and mortality in the transplant patient population, resulting in the dysfunction and destruction of the graft by the host's immune system via cellular or antibodymediated mechanisms. Acute cellular rejection (ACR) is more common and better characterized than antibody-mediated rejection, which to date lacks any widely agreed upon, standardized set of diagnostic criteria. We present a case of AMR attributable to a rare phenomenon, non-human leukocyte antigen (HLA) antibodies.

Case Report: A 50-year-old male underwent an uneventful single lung transplant for pulmonary sarcoidosis. Donor and recipient blood type was $A$ positive. No pretransplant donor-specific antibodies were identified. Flow cytometric crossmatch was negative. The postoperative course was significant for a single-unit transfusion of packed red blood cells on postoperative day (POD) 1 and persistent asymptomatic Serratia marcescens in bronchial washes despite ongoing levofloxacin treatment. A surveillance biopsy (POD 34) showed no evidence of rejection. One week later (on POD 41), the patient presented with fever, shortness of breath, and imaging abnormalities of the grafted lung. Inpatient antibiotic escalation to cefepime, ertapenem, and meropenem resolved the positive cultures and fever, but the patient's respiratory function continued to decline, requiring intubation and extracorporeal membrane oxygenation. High-dose steroids and therapeutic plasma exchanges were initiated for suspected acute rejection. During the workup, a newly developed anti-A1 red blood cell antibody was identified. Despite supportive efforts, the patient died on POD 55, 14 days after symptomatic presentation.

Conclusion: This case highlights the clinical significance of AMR in lung allografts, as well as the need to investigate both HLA and non-HLA antibody sources in pulmonary transplant rejection refractory to treatment.

Keywords: Fatal outcome, graft rejection, HLA antigens, lung transplantation

Address correspondence to Caroline R. Alquist, MD, PhD, Department of Pathology, Ochsner Clinic Foundation, 1514 Jefferson Hwy., New Orleans, LA 70121. Tel: (504) 842-5035. Email: caroline.alquist@ochsner.org

\section{INTRODUCTION}

Lung transplantation has been an essential component of therapy for end-stage lung disease since the early 1980s. Despite this longevity and advances in imaging, molecular identification platforms, and pharmaceuticals used to prevent graft rejection, the 5-year survival for posttransplant patients remains approximately $50 \% .{ }^{1}$ A major cause of morbidity and mortality in lung transplant recipients is the development of graft dysfunction or failure secondary to destruction by the host's immune system. Unlike other commonly transplanted solid organs, lungs are continually exposed to the outside environment and its pathogens. To combat these exposures, lungs are equipped with intrinsic immune-modulating cells that make the grafted tissue susceptible to immune activation and subsequent rejection. ${ }^{1}$
Acute rejection of lung allografts can occur days or even years after a successful transplant and plays a substantial role in the mortality of lung transplant recipients. In the year following their posttransplant hospital discharge, $29 \%$ of patients are treated for at least one episode of acute rejection. ${ }^{2}$ In addition, the frequency and severity of episodes of acute rejection represent a significant risk factor for the development of chronic airway rejection or bronchiolitis obliterans syndrome. ${ }^{2}$ The diagnosis of acute rejection is characterized according to the suspected underlying immune process causing damage to the graft, namely acute cellular rejection (ACR) or antibody-mediated rejection (AMR). ${ }^{2}$ ACR occurs more frequently than AMR, and the graft damage is mediated by recipient $T$ lymphocytes upon recognition and interaction with donor human leukocyte 
antigens (HLAs) or other antigens present in donor tissue. $^{2}$ Presensitization-or significant exposure prior to transplant-to an alloantigen that elicits an immune response with the resultant production of antibodies directed against that antigen is not uncommon. Evidence of presensitization can be found in $10 \%-15 \%$ of lung transplant recipients. $^{2,3}$ Although encountering donor-specific antibodies (DSAs) is relatively common, AMR has been relatively recently recognized compared to ACR. Not until 2016 did the Banff study seek to demonstrate histologic changes in lung grafts specific to patients with circulating DSAs. ${ }^{4}$ The damage occurs when recipient antibodies bind antigens in donor tissue, which in turn amplifies the immune response. ${ }^{2}$ DSAs can be preformed or can arise de novo and may be directed against HLAs or non-HLAs. We report a case of acute lung rejection attributable to AMR.

\section{CASE REPORT}

Following induction, a 50-year-old male received an uneventful single lung transplant for respiratory failure secondary to pulmonary sarcoidosis. Both donor and recipient blood types were group A positive. No pretransplant DSAs were present, and the virtual and retrospective crossmatches by flow cytometry were negative. The patient's initial postoperative course was significant for a single transfusion of packed red blood cells (pRBCs) on postoperative day (POD) 1, a hematoma evacuation on POD 2, and persistent Serratia marcescens-positive cultures from multiple bronchial washes despite antibiotic treatment guided by susceptibility studies. The patient was discharged home on POD 14.

Approximately 2 weeks after discharge (on POD 34), surveillance bronchoscopy was unremarkable, and a transbronchial biopsy obtained at that time showed no evidence of acute rejection, inflammation, or chronic airway rejection. Cultures obtained at that time continued to grow $S$ marcescens. Although the patient remained asymptomatic, he was started on $500 \mathrm{mg}$ levofloxacin daily.

On POD 41, the patient presented with fever, shortness of breath, nausea, and vomiting and was admitted to the hospital. Imaging revealed patchy consolidations and air bronchograms in the grafted lung, as well as a small pleural effusion. An infectious disease consult was initiated, and antibiotics were escalated throughout the patient's hospital course: from cefepime $2 \mathrm{~g}$ intravenously (IV) 3 times daily, to ertapenem $1 \mathrm{~g}$ IV daily, and then to meropenem $2 \mathrm{~g} \mathrm{IV} 3$ times daily. Despite resolution of his fever and the positive cultures, the patient's respiratory function continued to worsen. Additional lung biopsies were unobtainable because of the patient's anticoagulation status and a concern for pulmonary embolism.

No DSAs were identified by HLA antibody assays. Samples were sent to reference laboratories to detect other potentially causative non-HLA antibodies: antiperlecan, antimajor histocompatibility complex class I-related chain $A$ (MICA), anti-angiotensin II type 1 receptor (AT1R), anti-K $\alpha 1$ tubulin, and anti-type $V$ collagen. All assays returned negative. The patient became profoundly anemic and thrombocytopenic, necessitating the transfusion of 4 units of pRBCs and 1 unit of apheresis platelets on POD 48, 1 unit of pRBCs on POD 52, and 1 unit of apheresis platelets on POD 54. During routine pretransfusion testing on POD 52, a type and screen using gel column agglutination and confirmatory tube red cell agglutination grading revealed the presence of a newly formed anti-A1 antibody with a titer of 2 , reacting at $37^{\circ} \mathrm{C}$. High-dose steroids and a series of 3 single-volume therapeutic plasma exchanges were administered to treat the suspected AMR but resulted in no clinical improvement. The patient eventually required mechanical ventilation, followed by extracorporeal membrane oxygenation.

After a discussion with the family, the decision to withdraw care was made. The patient died on POD 55, 14 days after symptomatic presentation. The cause of death was listed as complications of lung transplantation, specifically AMR. No autopsy was performed according to the family's wishes. Additional subtype testing could not be performed because of the lack of appropriate samples. The Figure depicts the patient's clinical course.

\section{DISCUSSION}

AMR occurs when recipient antibodies, whether preformed or arising de novo, interact with cell surface antigens in the donor tissue. The damage incurred is the result of amplification of the immune response via complementdependent (cytotoxic) or complement-independent mechanisms. The majority of DSAs are formed against foreign HLAs in the grafted tissue, but antiendothelial antibodies and autoantibodies can also be involved in the destruction and accelerated dysfunction of grafted tissues. Additionally, antibodies directed against vimentin, collagen V, K $\alpha 1$ tubulin, AT1R, and MICA can be present before or after transplantation. $^{3}$ In our case, tests for donor-specific anti-HLA antibodies and the aforementioned non-HLA antibodies all returned negative results. The only positive finding in this case was the development of the non-HLA antibody anti-A1.

Unexpected $\mathrm{ABO}$ incompatibility playing a role in transplant rejection is exceedingly rare. $\mathrm{ABO}$ blood group phenotypes are determined by the enzymatic addition or lack of carbohydrate antigen deposited on a surface precursor molecule. Blood group $A$ is further divided into multiple subgroups, of which $\mathrm{A} 1$ and $\mathrm{A} 2$ are the most common. The difference between these subgroups is based largely on the functional properties of the individual's inherited enzyme. For example, subgroup $\mathrm{A} 1$ is present in $80 \%$ of those with $A$ or $A B$ blood types and possesses an enzyme 5 to 10 times more active than its $A 2$ counterpart. ${ }^{5,6}$ As a result of this enzymatic efficiency, individuals with the $A 1$ subgroup have increasingly complex branching oligosaccharides that display significantly more $A$ antigen on their cell surface compared to the A2 subgroup. ${ }^{5}$ Development of anti-A1 antibodies is seen in 1\%-8\% of previously sensitized individuals with the A2 subgroup and is usually of the immunoglobulin ( $\mathrm{lg}) \mathrm{M}$ isotype that tends to react best at or below room temperature and fails to cause hemolysis, placing the antibody in the realm of clinical insignificance. ${ }^{7}$ IgG isotypes are generally thought to be the most destructive, especially the subtypes with complement-binding abilities. ${ }^{2}$ Other factors that can influence the clinical significance of an antibody are binding affinity and antibody load.

In our case, both the donor and recipient were known to have group A blood, but only the donor had been further subclassed as type A1. Subclassification of transplant recipients is not routinely performed because of the rarity of 


\section{Single Lung Transplant Uneventful}

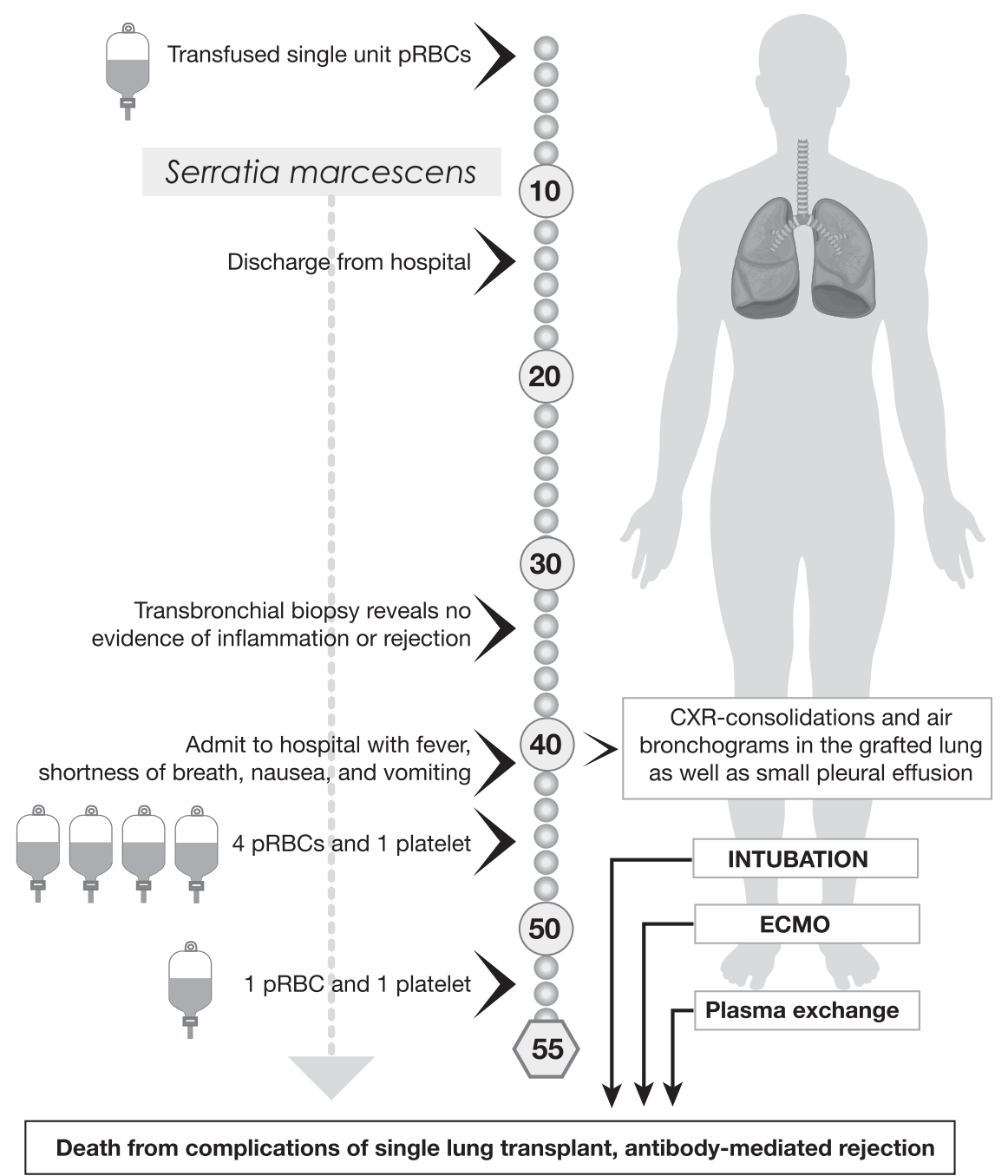

Figure. The patient's clinical course by postoperative day. CXR, chest $x$-ray; ECMO, extracorporeal membrane oxygenation; pRBCs, packed red blood cells.

developing clinically significant anti-A1 IgG antibodies. The anti-A1 antibody in our patient reacted at $37^{\circ} \mathrm{C}$ (normal body temperature), characteristic of a clinically significant antibody. Interestingly, our patient's antibody only had a titer of 2 and appeared late in his clinical course. We believe antibody levels were significantly lowered by graft adsorption because of the considerable endothelial surface area of the lung and may have masked the detection of this antibody. Given the late presentation of the anti-A1 antibody, we had to exclude the possibility of passive antibody transfer via temporally concordant group $O$ platelet transfusions during the patient's final week of treatment. A review of our data confirmed that both of these platelet products were from group A donors, rendering this possibility unlikely. Postmortem subtyping of the donor tissue would have helped clarify the diagnosis, but as stated in the case, additional testing was not performed given the lack of tissue availability.

We cannot completely exclude the possibility that the patient's persistent asymptomatic $S$ marcescens infection may have been a factor in his respiratory failure. Infection and pneumonia are common during the first year after lung transplantation because of environmental exposures and decreased mucociliary clearance and cough reflexes intrinsic to a denervated allograft. The presence of $S$ marcescens has been reported in approximately $2 \%-5 \%$ of posttransplant infections, but emerging data question the role of $S$ marcescens as a pathogen. ${ }^{8,9} S$ marcescens is commonly isolated from the sputum of patients with cystic fibrosis, but studies in this patient population have shown no change in mortality or lung function, even in patients who are chronically infected. ${ }^{10,11}$

\section{CONCLUSION}

After an extensive search of the literature, we were unable to find any similar case reports describing an adverse outcome of a single lung transplant that was attributed to the development of the anti-A1 antibody, suggesting that the development of a clinically significant anti-A1 antibody is exceedingly rare. The purpose of this report is to highlight the clinical significance of both HLA and non-HLA antibodies in acute pulmonary transplant rejection and to document an 
unusual case of non-HLA AMR associated with an $A B O$ anti-A1 antibody.

\section{ACKNOWLEDGMENTS}

The authors have no financial or proprietary interest in the subject matter of this article.

\section{REFERENCES}

1. Benzimra M, Glanville AR. Lung transplant rejection and surveillance in 2016: newer options. Curr Transpl Rep. 2016 Jun;3:192-198. doi: 10.1007/s40472-016-0104-y.

2. Roden AC, Aisner DL, Allen TC, et al. Diagnosis of acute cellular rejection and antibody-mediated rejection on lung transplant biopsies: a perspective from members of the pulmonary pathology society. Arch Pathol Lab Med. 2017 Mar;141(3):437-444. doi: 10.5858/arpa.2016-0459-SA.

3. Levine DJ, Glanville AR, Aboyoun C, et al. Antibody-mediated rejection of the lung: a consensus report of the International Society for Heart and Lung Transplantation. J Heart Lung Transplant. 2016 Apr;35(4):397-406. doi: 10.1016/j. healun.2016.01.1223.

4. Wallace WD, Li N, Andersen CB, et al. Banff study of pathologic changes in lung allograft biopsy specimens with donor-specific antibodies. J Heart Lung Transplant. 2016 Jan;35 (1):40-48. doi: 10.1016/j.healun.2015.08.021.
5. Hosoi E. Biological and clinical aspects of $A B O$ blood group system. J Med Invest. 2008 Aug;55(3-4):174-182.

6. Storry JR, Olsson ML. The ABO blood group system revisited: a review and update. Immunohematology. 2009;25(2):48-59.

7. Fadeyi EA, Stratta RJ, Farney AC, Pomper GJ. Successful ABOincompatible renal transplantation: blood group $\mathrm{A} 1 \mathrm{~B}$ donor into A2B recipient with anti-A1 isoagglutinins. Am J Clin Pathol. 2016 Aug;146(2):268-271. doi: 10.1093/ajcp/aqw101.

8. Campos S, Caramori M, Teixeira R, et al. Bacterial and fungal pneumonias after lung transplantation. Transplant Proc. 2008 Apr;40(3):822-824. doi: 10.1016/j.transproceed.2008.02.049.

9. Kramer MR, Marshall SE, Starnes VA, Gamberg P, Amitai Z, Theodore J. Infectious complications in heart-lung transplantation: analysis of 200 episodes. Arch Intern Med. 1993 Sep 13;153(17):2010-2016.

10. Belkarty B, Bilton D, Jones A. Serratia marcescens: an emerging pathogen or innocent bystander? J Cyst Fibros. 2015 Jun;14(1);S7260. doi: 10.1016/S1569-1993(15)30237-X.

11. Lata $C$, Parkins $M$, Somayaji $R$, et al. Epidemiology and clinical outcomes of Serratia marcescens infections in adults with cystic fibrosis. Open Forum Infect Dis. 2016 Dec;3(1):1228. doi:/10.1093/ofid/ofw172.931.

This article meets the Accreditation Council for Graduate Medical Education and the American Board of Medical Specialties Maintenance of Certification competencies for Patient Care and Medical Knowledge. 